\title{
A novel wearable measurement system for ambulatory assessment of joint loading in the occupational setting
}

\author{
Gert Faber $^{* a, b}$, Chien-Chi Chang ${ }^{\mathrm{c}}$, Idsart Kingma ${ }^{\mathrm{b}}$, Jack T. Dennerlein ${ }^{\mathrm{a}}$ \\ ${ }^{a}$ Department of Environmental Health, Harvard School of Public Health, 665 Huntington Avenue, Boston, MA \\ 02115, USA \\ ${ }^{b}$ Research Institute MOVE, Faculty of Human Movement Sciences, VU University, Van der Boechorststraat 9 , \\ 1081 BT, Amsterdam, The Netherlands \\ ${ }^{c}$ Liberty Mutual Research Institute for Safety, 71 Frankland Road, Hopkinton, MA 01748, USA
}

\begin{abstract}
It is know that biomechanical overexposure of the joints is an important cause of occupational injuries. This paper presents a novel wearable measurement system for automated assessment of joint loading in the occupational setting. The wearable measurement system consists of a full body inertial sensor motion capture system which can be worn under the clothes and shoes instrumented with 3D force sensors (ForceShoes). Promising results have been found for the performance of the inertial sensor system and the ForceShoe, separately. Validation experiments are in preparation in which the performance of the combined measurements system will be tested in the laboratory by comparing the assessed joint loading to the joint loading assessed by a conventional state-of-the-art lab-based method.
\end{abstract}

Keywords: Ambulatory/Wearable measurement system, Occupational biomechanics, ForceShoes, Inertial sensors.

\section{Introduction}

Biomechanical overexposure of the joints is an important cause of occupational injuries [1] and thus it is important to evaluate the biomechanical exposure in the workplace. Currently available methods, such as video analysis, are, however, time-consuming and therefore not frequently applied. Hence, an easier applicable method to assess joint loading in the field is desirable.

This paper presents a novel wearable measurement system for automatic and continuous ambulatory assessment of joint loading in the occupational setting.

\section{Practice innovation}

The wearable measurement system (Xsens Technologies, Netherlands, Figure 1) consists of a full body inertial sensor motion capture system (measuring segment orientation) which can be worn under the clothes and shoes instrumented with 3D force sensors (ForceShoes). Using this system you can not only assess joint loading due to body postures, but also the joint loading due to external loads (using the forces measured by the ForceShoes), occurring for example during manual materials handling.

${ }^{*}$ Corresponding author. E-mail: gertfaber.sci@gmail.com, Tel no.: +1-617-384-8768 


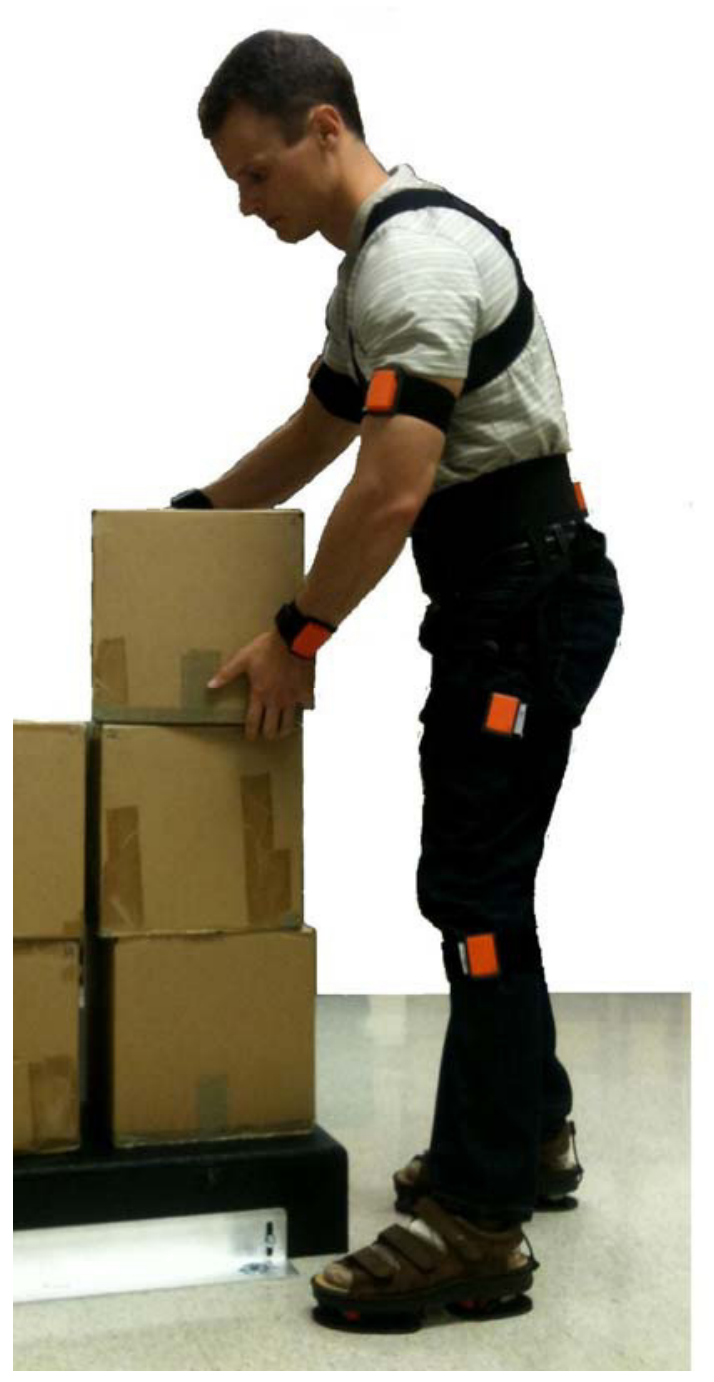

Fig 1. The wearable Xsens measurement system consisting of a full body inertial sensor motion capture system and shoes instrumented with 3D force sensors (ForceShoes). Note that the inertial sensors (the orange sensors) can be worn under the clothes as well.

\section{Findings}

Recent studies performed by our group have investigated the performance the separate components of the whole measurement system.

In one study [2] the optimal inertial sensor location for the measurement of trunk inclination was investigated. The optimal location was found to be at about $25 \%$ of the distance from the sacrum to the $7^{\text {th }}$ cervical processus spinosus. At this location the RMS error in trunk inclination was around $5^{\circ}$.

A following study [3] tested the performance of ForceShoes in measuring 3D ground reaction forces (GRFs) during common work tasks. Compared to force plate measurements small errors were found: GRF error $<3 \%$ and Center of pressure error $<10$ $\mathrm{mm}$.

A third study [4] assessed the effect of using orientation sensors (like inertial sensors) instead of position sensors in a bottom-up inverse dynamic analysis of joint loading. Knee moments could be assessed with good accuracy (errors $<4 \%$ ) and Hip and L5/S1 moments with moderate accuracy (errors $<14 \%$ ).

\section{Discussion}

Currently we are validating the combined measurement system in the laboratory by comparing the assessed joint loading to that of a conventional state-of-the-art lab-based method [5]. Both a bottomup (starting at the feet) and a top-down (starting at the hands) inverse dynamics model will be considered. If the proposed system proves to be a valid measurement system, we will conduct feasibility field studies assessing the capabilities and limitations in real work environments.

\section{References}

[1] da Costa BR \& Vieira ER. Risk factors for work-related musculoskeletal disorders: A systematic review of recent longitudinal studies. Am. J. Ind. Med. (2010), 53(3), 285-323.

[2] Faber GS, Kingma I, Bruijn SM \& van Dieën JH. Optimal inertial sensor location for ambulatory measurement of trunk inclination. J. Biomech., 2009, 42(14): 2406-2409.

[3] Faber GS, Kingma I, Martin Schepers H, Veltink PH \& van Dieen JH. Determination of joint moments with instrumented force shoes in a variety of tasks. J. Biomech., 2009, 43(14): 2848-54.

[4] Faber GS, Kingma I \& van Dieën JH. Bottom-up estimation of joint moments during manual lifting using orientation sensors instead of position sensors. J. Biomech., 2010, 43(7): 1432-6.

[5] Kingma I, de Looze MP, Toussaint HM, Klijnsma HG \& Bruijnen TBM. Validation of a full body 3-D dynamic linked segment model. Hum. Mov. Sci. (1996), 15(6), 833-860. 